Voix et Images

volxetimages

\title{
Modernité/Postmodernité : problème taxinomique? combat de légitimité? crise de culture?
}

\section{François Gallays}

Volume 14, numéro 3 (42), printemps 1989

Gabrielle Roy

URI : https://id.erudit.org/iderudit/200803ar

DOI : https://doi.org/10.7202/200803ar

Aller au sommaire du numéro

Éditeur(s)

Université du Québec à Montréal

ISSN

0318-9201 (imprimé)

1705-933X (numérique)

Découvrir la revue

Citer cet article

Gallays, F. (1989). Modernité/Postmodernité : problème taxinomique? combat de légitimité? crise de culture? Voix et Images, 14(3), 504-507.

https://doi.org/10.7202/200803ar d'utilisation que vous pouvez consulter en ligne.

https://apropos.erudit.org/fr/usagers/politique-dutilisation/ 


\section{Modernité/Postmodernité: problème taxinomique? combat de légitimité? crise de culture?}

\section{par François Gallays, Université d'Ottawa}

Si pour le philosophe Gianni Vatimo (1987) la postmodernité signale la fin de toute pensée dogmatique et l'avènement d'une pensée souple ou «faible», dont la seule ressource serait désormais la mobilité de l'interprétation, au Québec dans deux livres récents, ce «mot-concept» se fait tantôt qualifier de simple étiquetage ou de pensée réactionnaire, tantôt afficher comme bannière pour un nouveau type de roman ou arborer comme pertuisane dans le combat qu'on se livre au sein de l'institution littéraire. Bref, on frôle le grand désordre conceptuel. Par ailleurs, Vatimo, lui, n'y verrait peut-être pas autre chose qu'une certaine mobilité de l'interprétation.

De cette manière, on renouvellera continuellement l'air de la machine, le soufflet chassant celui qui y est, et obligeant celui de dehors d' entrer par les tuyaux. (Gravesande, Usage de la chambre obscure)

J'ai cru bon de citer ce texte que Pierre Milot a choisi de placer en exergue car, en peu de mots, il éclaire, avec un brin d'humour me semble-t-il, le choix du titre: la Camera obscura du postmodernisme1, lequel est emprunté à un des chapitres, le quatrième des six qui composent cet ouvrage et dont seul le dernier, «Modernité, avant-garde, postmodernité», n'aura pas été précédemment publié dans une revue sous forme d'article.

Maniant avec maestria les concepts opératoires d'inspiration économique que propose le sociologue Pierre Bourdieu dans son ouvrage désormais célèbre, la Distinction (1979), pour rendre compte du fonctionnement du champ culturel, Pierre Milot analyse dans les cinq derniers chapitres le comportement d'agents culturels divers: les revues la Nouvelle Barre du jour et les Herbes rouges (chapitre I), des écrivains/écrivants: Jean Larose (chapitre II), Claude Beausoleil, André Beaudet (chapitres III et IV), Michel Muir (chapitre III), Pierre Bertrand (chapitre IV), Hugues Corriveau et Normand de Bellefeuille (chapitre VI).

De ces cinq premiers chapitres, le premier, celui qui porte sur les deux revues d'avant-garde, me semble de loin le plus pertinent. Non pas que les quatre suivants soient dénués d'intérêt. Par exemple, l'administration d'une leçon de méthode à un Jean Larose qui, à l'analyse rigoureuse, a parfois préféré la formule bien frappée et la métaphore bien trouvée a quelque chose de tonifiant. Par contre, lorsque Milot propose une analyse de nature sociologique des interventions d'André Beaudet dans le champ littéraire de l'avant-garde, le lecteur (que je suis), s'il acquiesce à la démonstration du critique, ne peut toutefois s'empêcher, mais à tort sans doute, de penser, ne serait-ce que très fugitivement, qu'il s'exerce là simultanément un petit règlement de compte. 
Le modèle sociologique affiche, selon moi, un meilleur rendement lorsqu'il est employé à analyser le comportement d'agents collectifs comme les deux revues d'avant-garde (BJ/NBJ et HR), car les motivations sont de façon générale clairement exprimées, les ambitions avouées sans vergogne et les enjeux affichés. Toujours est-il que ce premier chapitre, «L'avant-garde: processus institutionnel et conflits de légitimité», par l'analyse des discours, des remarques et des déclarations de divers agents liés étroitement à ces deux revues, démonte le processus par lequel l'avant-garde québécoise des années soixante-dix est montée à l'assaut de la légitimité.

Au départ subversive, l'avant-garde littéraire, se donnant comme gonfalons le formalisme, la nouvelle écriture, revendiquera avec fierté comme espace à occuper les marges du champ littéraire et se donnera comme agents de distinction les collaborateurs de la revue française Tel Quel. Mais par la suite, accompagné d'une opération de reconversion grâce à quoi sont reniées les anciennes positions au profit de nouvelles qui se traduiront par des expressions telles que nouveau lyrisme ou nouvelle lisibilité ou, encore, retour du sacré, un travail d'institution s'effectue qui fera passer les agents actifs dans la Nouvelle Barre du jour et dans les Herbes rouges des marges de l'institution littéraire à son centre.

Dans le sixième chapitre, Milot s'efforce de distinguer soigneusement ce qui constitue une avant-garde par opposition à la modernité, d'une part, et la postmodernité par rapport à la modernité, d'autre part. Pour ceux qui ont l'habitude de voir ou d'entendre ces mots employés sans que leur sens soit toujours précisé, ce texte pourrait servir de guide. Mettant en scène trois acteurs principaux: Jürgen Habermas, Jean-François Lyotard et Guy Scarpetta, le premier, défenseur de la modemité, et les deux autres, promoteurs de la postmodernité, Milot, par l'entremise d'une citation empruntée à Peter Bürger, propose de comprendre la modernité et l' avant-garde comme deux orientations différentes $[\ldots]$, la modernité correspond a 1789 et l'avant-garde d 1793, cette dernière représentant une radicalisation da la fois logique et aporétique de la modernité. Habermas, quant à lui, considère l'avant-garde comme faux dépassement du projet de la modernité. Fort de cette distinction, Milot est d'avis que Scarpetta devrait parler de post-avant-gardisme plutôt que de postmodernisme, puisque ce dernier entretient dans ses propos une certaine confusion entre l'avant-garde, la modernité et le modernisme: L'idéologie avant-gardiste (moderniste) a d' une certaine façon épuisé sa fonction. Pour Lyotard, le postmoderne ne se situe pas après le moderne, ni contre lui. Il y était déjà inclus, mais caché. Aussi Scarpetta refoule Lyotard dans la stricte modernité alors que Habermas voit en lui un néo-conservateur et son postmodernisme comme l'expression d'une position néo-conservatrice, car il fait de la théorie critique une activité délégitimée et de la démystification théorique une illusion.

Le débat opposant Habermas et Lyotard (et Scarpetta), dont fait état Pierre Milot, débouche nécessairement selon celui-ci sur le problème de l'autonomie du champ philosophique, problème qui oppose la philosophie fondée sur le rationalisme argumentatif à ce que José-Guilherme Merquior appelle la littéro philosophie au style d'une haute tenue littéraire, que pratiquent des «penseurs» 
comme Bergson, Foucault, Bataille, Blanchot, mais dont le contenu est trop souvent obscur ou, du moins, difficile à cerner avec précision.

Voilà un petit livre qui donne à réfléchir et qui vaudra à son auteur, j'en suis persuadé, une sensible augmentation de son capital symbolique!

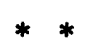

Comprenant les actes d'un colloque qui eut lieu au Centre d'études canadiennes de l'Université libre de Bruxelles (1985), Modernité/Postmodernité du roman contemporain de Québec et d'Acadie ${ }^{2}$ porte les marques de son origine, c'est-à-dire que, malgré le titre qui se veut «rassembleur», les articles rassemblés ici présentent une bien grande diversité. Sont étudiés entre autres le thème de l'amour, les lieux de plaisir, le thème de la creation impossible, le discours surréaliste chez les romanciers québécois, les jeux de mots. Nombre de romanciers font l'objet d'une étude: Nicole Brossard, Hubert Aquin, Michel Tremblay, Jacques Godbout, Réjean Ducharme, Germaine Comeau et Antonine Maillet. Et bien que le colloque ait eu comme thème la modernité et la postmodemité, ce ne sont pas tous les textes, tant s'en faut, qui s'y reportent. De plus, parmi ceux qui s'y reportent, aucun n'abordera le problème épistémologique que soulève la formulation particulière du titre Modernité/Postmodernité... Patrick Imbert, dans son article «Identité et présence du discours surréaliste chez les romanciers québécois modernes», y fait bien allusion lorsqu'il affirme qu'une certaine critique notamment celle qui distribue allègrement l'étiquette «moderne» ou "postmoderne» sans distinction, tente d'enfermer l'art dans un "cosmopolitisme qui n'est pas nouveau mais que l'on valorise depuis quelque temps» (G. Scarpetta, Éloge du cosmopolitisme, 1981)... Ce cosmopolitisme qu'Imbert qualifie de pseudo-non-territorialité marchande est bien autre chose, ajoute-t-il, que la différence qui est dissidence, que l'on retrouve dans certains romans de la littérature québécoise, dont ceux de Jacques Brossard, Aquin, Ducharme et Ferron, textes qui participeraient à la modernité au $\mathrm{XX}^{\mathrm{e}}$ siècle. Mais qu'est-ce au juste que la modernité pour Imbert? Elle est d'abord une façon d'être, un mode d'agir plutôt, car elle est inscrite dans un présent, un advenant permanent [qui] est aussi un point de fuite, $[. .$.$] intensité de$ simultanéités..., dans lesquels le sujet est à redéfinir comme point vide et où [i]l $n^{\prime} y$ a plus d'origine, [...] mais accélération [...] des valeurs dans un présent où l'identité se fonde/se fond dans la présence des autres en soi [...].

Évidemment, cela était écrit en 1985 et nous sommes maintenant en 1989. Quatre ans, c'est long pour quiconque conçoit le temps comme emballement du présent, c'est suffisamment long pour que se soient opérées dans cet entre-deux au moins une ou deux révolutions et ce, simultanément..., car cette importance démesurée accordée au présent privilégie, il me semble de façon exorbitante, la toute dernière mode ou, comme qui dirait, l'extrême contemporain.

Si Imbert voit dans Aquin et Ducharme deux romanciers chez qui s'exprime la modernité, d'autres voient en eux des représentants de la postmodernité. Ainsi 
en va-t-il de Franca Marcato Falzoni qui, dans son excellent article sur la Fille de Christophe Colomb de Réjean Ducharme, déclare dès l'incipit que $[l] a$ modernité, entendue comme valeur négative, est un thème que l'on retrouve dans toute l' œuvre de Ducharme pour qui le présent est une ère de décadence et le monde actuel peuplé d'ex-géants devenus des nains. Aussi voit-elle dans la Fille de Christophe Colomb le récit de l'instauration de la modernité avec meurtre / transgression consciente / inconsciente et comment elle est punie par une Postmodernité ingénue et ingénument reliée à la Prémodernité.

Janet Paterson, pour sa part, après avoir proposé ce qu'elle considère comme les traits principaux de la définition de l'écriture postmoderne (pluralité et fragmentation du sujet de l'énonciation; surcodage de la fonction du narrataire; importance accordée à l'écriture et à la rupture; caractérisation, au niveau du code, par l'autoreprésentation et par l'intertextualité) Janet Paterson, dis-je, entreprend l'examen de Trou de mémoire de Hubert Aquin à la suite duquel on peut, si les critères retenus sont justes, induire que ce roman s'inscrit bien davantage dans le sillage de la postmodernité que dans celui de la modernité. Enfin, à signaler l'article «Nicole Brossard: une influence coûteuse» où Louise Milot, au nom d'une modernité bafouée, d'une nouvelle écriture abandonnée, déplore que Nicole Brossard, à partir de 1977, date de parution de son roman l'Amèr, ait tourné le dos à l'écriture autoréférentielle, pour aborder une littérature au contenu féministe de plus en plus manifeste. L'auteur de cet article affirme donc qu'il s'est effectué chez Nicole Brossard un repliement sur une position nettement moins moderniste lorsque ses textes cessent d'exploiter le potentiel de la matérialité du texte pour explorer les arcanes du féminisme. La position critique qu'épouse Louise Milot dans son article ne laisse d'étonner quelque peu, car elle constitue en entité, en une sorte d'objet autonome, ce qui n'est toujours qu'une pratique personnelle, c'est-à-dire un projet. Ne participant à aucune essence préalable, ce qu'on pourrait appeler le «potentiel» de cette nouvelle écriture ne peut jamais, me semble-t-il, être autre chose que le résultat d'un jugement fondé sur la rétrospection.

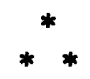

Deux livres, deux sujets communs: la modernité et la postmodernité. La lecture de l'un donne parfois l'impression d'un trop grand éparpillement. L'autre, celui de Pierre Milot, même s'il n'est pas exclusivement consacré à ce couple antagoniste, ne s'en éloigne toutefois jamais très loin, de sorte que le lecteur reste avec l'impression d'avoir acquis une idée relativement claire quant à la nature de ces deux concepts et quant à leur fortune au sein de l'institution littéraire du Québec. Ce n'est pas une impression désagréable...

1 Pierre Milot, la Camera obscura du postmodernisme, Montréal, l'Hexagone, 1988, 91 p. (Essais littéraires).

2 Jacques Allard et Madeleine Frédéric (sous la direction de), Modernité/Postmodernité du roman contemporain de Québec et d'Acadie, Montréal, UQAM, 1987, 200 p. (les Cahiers du Département d'études littéraires, no 11). 